\title{
Aspectos Matemáticos e Estatísticos da Lei de Mitscherlich
}

Tese apresentada - aprovada na Ila. Reuniño Brasileira de Ciôncia do Solo, realizada om Campinas, Eotado de São Paulo, de 11 a 23 de Julho de 1949.

\section{FREDERICO PIMENTEL GOMES}

Assistente e Livre-docente da cadeira de Matemática da Escola Superior de Agricultura "Luiz de Queiroz"

EURTPEDES MALAVOLTA

Assistente da Seç̧ão de Qúmica Agrícola da Escola Superior de Agricultura "Luiz de Queiroz"

\section{INDICE}

1 -Introdução ......................... 194

2 - 0 problema a resolver ................. 194

3-O cálculo dos parâmetros da primeira aproximação de Mitscherlich ...................... 200

4 - Um exemplo de aplicação do método dos momentos 205

5 - Ainda o método dos momentos . ........... 211

6 - Um mau exemplo da literatura ............... 212

7 - A técnica das experiênecias em vasos ......... 215

8 - A interpretação dos resultados $\ldots \ldots \ldots \ldots \ldots \ldots 218$

9 - Comentários sôbre as experiências em vasos ..... 219

10 - Conelusóes ............................. 221

11 - Abstract . . . . . . . . . . . . . . . . . . . . . . . . . 223

12 - Bibliografia consultada................... 227 


\section{1 - INTRODUÇÃO}

Este trabalho tem por fim discutir alguns aspectos da lei de MITSCHERLICH, aspectos êsses que nos parecem dignos de um estudo mais aprofundado.

Todos sabem que a lei de MITSCHERLICH tem contra si muitas objeções. Mas a seu favor há uma série enorme de pesquisas e de bons resultados obtidos em experiências em vasos ou no campo, inclusive algumas realizadas entre nós (1).

Os autores já abordaram o assunto em trabalho anterior (2). Aqui, porém, partirão de um ponto de vista mais geral, procurando encarar o problema de uma maneira bem de acôrdo com o rigor científico atual. Isso se faz necessário, pois a literatura sôbre o assunto, na sua maior parte esparsa em revistas agronômicas alemãs, peca muito quanto ao rigor e está cheia de controvérsias violentas, nem sempre bem fundadas.

\section{2 - O PROBLEMA A RESOLVER}

Feita uma experiência de adubação com doses crescentes de um determinado adubo, obtém-se uma série de dados, como a do quadro seguinte, onde se supõem 4 repetições.

\section{PRODUÇAOO EM QUINTAIS-METRICOS POR HECTARE}

\begin{tabular}{c|c|c|c|c|c}
\hline $\begin{array}{c}\text { Quintais/ha } \\
\text { P2O5 }\end{array}$ & 0 & 0,2 & 0,5 & 0,8 & 1,2 \\
\hline \multirow{5}{*}{} & 20,5 & 22,0 & 24,0 & 27,1 & 27,9 \\
& 19,9 & 23,1 & 24,3 & 26,7 & 29,0 \\
& 20,0 & 21,9 & 26,4 & 26,2 & 28,3 \\
& 20,1 & 22,6 & 25,7 & 26,8 & 27,6 \\
\hline \multirow{2}{*}{ Médias } & 20,1 & 22,4 & 25,1 & 26,7 & 28,1 \\
\hline
\end{tabular}


Esses dados podem ser representados em um gráfico. $O$ problema a resolver consiste em interpolar uma curva que se adapte o melhor possível aos dados experimentais e que nos permita deduzir qual a quantidade de $\mathrm{P} 2 \mathrm{O}$ ex existente no solo à disposição da planta e qual a que se deve adicionar para obter o maior rendimento econômico possível. A curva em questão deve apresentar os seguintes característicos:

I - Deve adaptar-se, pelo menos razoàvelmente, a qualquer experiência análoga com qualquer planta cultivada.

II - Sua equação deve depender de poucos parâmetros fáceis de determinar.

Ora, há inúmeras funções à nossa escolha capazes de satisfazer a essas condições. Poderíamos utilizar, por exemplo, a análise harmônica e obter uma função interpoladora do tipo

$f(x)=\frac{a o}{2}+a 1 \cos x+a 2 \cos 2 x+\ldots+$

$+a n \cos n x+b 1 \operatorname{sen} x+b 2 \operatorname{sen} 2 x+\ldots+b n \cos n x$

tal como se costuma fazer em numerosos problemas de engenharia. Êsse processo foi utilizado por RIPPEL e MEYER (3) REDDIK e MILLER (4) dão os detalhes do cálculo algébrico.

Poderíamos também usar como função interpoladora uma série de polinômios de Legendre, o que também foi tentado por RIPPEL e MEYER (3). E poderíamos ainda considerar um polinômio de grau $n$ :

$$
P(x)=a o+a 1 x+22 x^{2}+\ldots+a n x^{n}
$$

e fazer sua interpolação pelos métodos estatísticos dados por FISHER (5, pp. 133-139) ou por RODRIGUES (6, pp. 363-366). Tal seria um procedimento mais de acôrdo com os conhecimentos modernos de Estatística e já preconizado em trabalhos antigos como os de FROHLICH (7) e o de PFEIFFER e outros (8).

A desvantagem, porém, dêsses e de outros métodos de interpolação está na arbitrariedade da função interpoladora. Essa arbitrariedade só pode ser levantada por estudos de natureza teórica, que nos levem a admitir como mais plausível um determinado tipo de função.

Ora, MITSCHERLICH (9) admitiu como razoável que, sendo $y$ a produção da planta, $x$ a quantidade fertilizante assimilável à sua disposição e $A$ uma constante, teríamos : 
$(2,01) \quad \frac{d y}{d x}=k(A-y)$,

onde $k$ é uma constante positiva.

A integração dessa equação, que foi discutida com detalhes no nosso trabalho anterior (2), nos dá como resultado:

$$
-\mathrm{L}(\mathrm{A}-\mathrm{y})=\mathrm{K}+\mathrm{kx} \text {, }
$$

onde $\mathrm{K}$ é a constante de integração e o símbolo $\mathrm{L}$ indica logarítmo natural ou neperiano. Fixado $\mathrm{x}=0$, é razoável admitir que temos $\mathrm{y}=\mathrm{o}$ e vem :

$$
\mathrm{K}=-\mathrm{LA} \text {. }
$$

Segue-se que :

$$
\mathrm{L}(\mathrm{A}-\mathrm{y})=\mathrm{LA}-\mathrm{kx} \text {. }
$$

Suporemos $x$ sempre positivo ou nulo.

Mas a quantidade de fertilizante à disposição da planta compõe-se de uma quantidade $b$ prèviamente existente no solo mais uma quantidade $\mathbf{x}$ de fertilizante que lhe foi adicionada. Obtemos assim a equação:

$$
\mathrm{L}(\mathrm{A}-\mathrm{y})=\mathrm{LA}-\mathrm{k}\left(\mathrm{b}+\mathrm{x}^{\prime}\right)
$$

ou, mais simplesmente,

$$
\mathrm{L}(\mathrm{A}-\mathrm{y})=\mathrm{LA}-\mathrm{k}(\mathrm{b}+\mathrm{x}) .
$$

Mas, como se sabe,

$$
L(A-y)=\frac{\log (A-y)}{\log e}, L A=\frac{\log A}{\log e}
$$

onde a abreviatura log indica logarítmo decimal e $e$, que vale aproximadamente 2,71828 , é a base do sistema neperiano. Segue-se que temos em logarítmos decimais :

$$
\log (\mathrm{A}-\mathrm{y})=\log \mathrm{A}-\mathrm{c}(\mathrm{x}+\mathrm{b}),
$$

onde $\mathrm{c}=\mathrm{k} \cdot \log \mathrm{e} \sim 0,4343 \mathrm{k}$ é uma nova constante.

De $(2,03)$ deduzimos logo que: 


$$
\log \left(1-\frac{y}{A}\right)=-c(x+b)
$$

Daí tiramos fàcilmente :

$$
y=A \quad\left[1-10^{-c(x+b)}\right]
$$

Como o segundo têrmo da expressão entre colchêtes é sempre negativo, segue-se que temos para qualquer valor de $x$ (finito) $\mathrm{y}<\mathrm{A}$. Mas se $\mathrm{x} \longrightarrow \infty$ vê-se logo que $\mathrm{y} \longrightarrow \mathrm{A}$. O parâmetro $A$ representa, portanto, uma produção máxima que seria atingida só para $x=\infty$.

Como temos sempre y $<$ A para qualquer valor (finito) de $x$, resulta que $\mathrm{A}-\mathrm{y}$ é positivo e portanto de $(2,01)$ resul-

ta que temos sempre $\frac{d y}{d x}>0$, isto é, que $y$ é função cres-

cente de $x$, como se devia esperar. $(2,01)$ nos mostra ainda que $k$ (e portanto também $c$, que lhe é proporcional) mede o efeito do adubo sôbre o aumento da produção. Por isso $c$ recebeu a denominação de valor ou fator ou coeficiente de eficácia.

Vemos que a hipótese de MITSCHERLICH nos conduz à função de $(2,05)$, cuja equação inclui apenas três parâmetros, todos três de significação objetiva relevante. Além disso numerosas experiências demonstram que na maioria dos casos essa função relativamente simples e com um número tão pequeno de parâmetros nos dá uma curva que se adapta razoàvelmente bem aos dados obtidos com qualquer planta e em qualquer solo. E ainda a função supõe sempre uma produção máxima inatingivel, o que é bem razoável.

Há, porém, como objeção mais importante, o fato seguinte. Verifica-se experimentalmente que, à medida que crescem as doses de adubo, cresce a produção até certo ponto, para logo começar a decrescer, chegando a anular-se quando a quantidade de adubo utilizada se torna excessiva. Ora, êsse fato importantíssimo não é considerado na equação $(2,05)$, que não pode representá-lo. Essa objeção levou MITSCHERLICH a tomar $(2,05)$ apenas como uma primeira aproximação da curva procurada e a adotar como uma segunda e mais perfeita aproximação a curva de equação.

$$
\text { 2,06) } \quad \mathrm{y}=\mathrm{A}[1-10-\mathrm{c}(\mathrm{x}+\mathrm{b})] 10-\mathrm{s} \times 2
$$


onde $s$ é o "fator de prejuizo" ("Schädigungsfaktor", em alemão). A equação diferencial correspondente é :

$$
(2,07) \quad \frac{\log e}{y} \cdot \frac{d y}{d x}=k \frac{10-c(x+b)}{1-10-c(x+b)}-2 s x \text {. }
$$

Não podemos deixar de observar aqui que RUSSELL (10, p. 140) dá a equação $(2,07)$ sob a forma

$$
\frac{1}{y} \quad \frac{d y}{d x}=c \frac{A-y}{y}-2 s x,
$$

afirmando que $(2,08)$ dá por integração $(2,06)$. E' fácil verificar o êrro dessa afirmativa, pois a função dada em $(2,06)$ absolutamente não satisfaz $(2,08)$.

Esse êrro aparentemente decorre de um estudo descuidado do artigo (11, p. 276) em que MITSCHERLICH apresentou sua segunda aproximação para a lei de eficácia. De fato, partindo da equação

$$
(2,09) \quad y=A(1-10-c x),
$$

onde $x$ está substituindo $\circ \mathrm{x}+\mathrm{b}$ usual, obteve por derivação

$$
\begin{aligned}
& \text { 2,10) } \frac{d y}{d x}=c . A 10-c x \text { L } 10 \\
& \text { Mas } \quad \text { L } 10=\frac{\log 10}{\log e}=\frac{1}{\log e} \cdot \quad \text { Logo: } \\
& \frac{d y}{d x}=c 1 \text { A. } 10-c x=c 1(A-y)
\end{aligned}
$$$$
\text { onde } \quad c 1=\frac{c}{\log e} \cdot \text { Daí resultou }
$$$$
\text { (2,11) } \quad \frac{1}{y} \cdot \frac{d y}{d x}=c 1 \frac{A-y}{y}
$$ 
Substituindo então y pelo seu valor dado em $(2,09)$ MITSCHERLICH obteve enfim a equação

2,12) $\frac{1}{y} \frac{d y}{d x}=c 1 \frac{10-c x}{1-10-c x}$.

Considerou então mais um têrmo $-2 k 1 \times$ nessa equação diferencial e escreveu :

$$
\frac{1}{y} \frac{d y}{d x}=c 1 \frac{10-c x}{1-10-c x} \cdot-2 k 1 x,
$$

onde $\mathrm{k} 1$ corresponde a $\frac{\mathrm{s}}{\log \mathrm{e}}$, na notação que utilizamos atrás. Da última equação foi que MITSCHERLICH obteve

$$
\int \frac{d y}{y}=\int c 1 \frac{10-c x}{1-10-c x} d x-\int 2 k 1 x d x
$$

Daí provém, depois de efetuada a integração, a equação

$$
\mathrm{L} y=\mathrm{L}(1-10-\mathrm{cx})-\mathrm{k} 1 \mathrm{x}^{2}+\mathrm{C} .
$$

Multiplicando-se tudo por log e chega-se logo à igualdade

$$
\log \mathrm{y}=\log (1-10-\mathrm{cx})-\mathbf{s x ^ { 2 }}+\mathrm{C},
$$

de onde se obtém

ou

$$
y=10 \mathrm{C}(1-10-\mathrm{cx}) 10-\mathrm{sx} 2 \text {, }
$$

$$
\mathrm{y}=\mathrm{A}(1-10-\mathrm{cx}) 10-\mathrm{sx2} \text {. }
$$

Note-se que nesta equação A já não representa a produção máxima.

Ora, até $(2,11)$ era indiferente escrever

$$
\frac{1}{y} \frac{d y}{d x}=c 1 \frac{A-y}{y}
$$


ou:

$$
\frac{1}{y} \frac{d y}{d x}=c 1 \frac{10-c x}{1-10-c x} .
$$

Juntando-se mais um têrmo $-2 \mathrm{k1} \times \mathrm{x}$ à segunda dessas equações, como fez MITSCHERLICH, automàticamente muda o valor de $y$, que passa a ser:

$$
y=A .(1-10-c x) e^{s \times 2} \text {. }
$$

Portanto não é indiferente como supôs RUSSELL, acrescentar o novo têrmo a $(2,11)$ ou $(2,12)$.

E' bem verdade que $(2,06)$ se adapta melhor que $(2,05)$ aos dados colhidos em experiências de adubação. Mas tem o defeito de ser uma função muito mais complicada e com um parâmetro a mais. Além disso o efeito do fator 10 - $\mathrm{sx2}$ só começa a aparecer para valores relativamente grandes de $x$, isto é, para adubações excessivamente pesadas. Por isso, excluidos os casos extremos, é sempre preferível utilizar a "primeira aproximação", expressa por $(2,05)$.

\section{3 - O CÁLCULO DOS PARAMMETROS DA PRIMEIRA}

\section{APROXIMAÇAOO DE MITSCHERLICH}

Admitida a fórmula de MITSCHERLICH :

$$
\mathrm{y}=\mathrm{A}[1-10-c(x+b)],
$$

suponhamos que dispomos dos dados de colheita yi $(=1,2, \ldots$, n) correspondentes às doses de fertilizante xi $(i=1,2 \ldots, n)$ e que queremos calcular os parâmetros $A, b, c$, para êsse caso. Um método que logo ocorre para realizar êsse cálculo é o dos quadrados mínimos, que, aliás, é sugerido por NIKLAS e MILLER (12).

A aplicação dêsse método ao caso seria feita como se segue. Uma vez calculados os parâmetros procurados teremos para cada dose xi de adubo uma produção observada yi e uma produção calculada A $[1-10 \multimap(x i+b)]$. Seja : 
Aspectos matemáticos e estatisticos

201

$$
\begin{aligned}
& z=\sum_{1=1}^{n}\left\{y_{1}-A\left[1-10^{-c\left(x_{1}+b\right)}\right]\right\} 2 \\
& \text { O método en estudo exige que tenhamos } \\
& \frac{d_{z}}{\partial A}=\frac{d_{z}}{\partial c}=\frac{d_{z}}{\partial b}=0 \text {. }
\end{aligned}
$$

Com o auxílio dessas três equações devemos calcular $A$, $b$, c. Podemos facilmente demonstrar que o sistema anterior é equivalente ao seguinte:

$$
\left\{\begin{array}{l}
\left.\sum: 2\right)\left\{y_{1}-A\left[1-10^{-c\left(x_{1}+b\right)}\right]\right\}=0 \\
\sum\left\{y_{1}-A\left[1-10^{-c\left(x_{1}+b\right)}\right]\right\} 10^{-c x_{1}} x_{1}=0 \\
\sum\left\{y_{1}-A\left[1-10^{-c\left(x_{1}+b\right)}\right]\right\} 10^{-c} x_{1}=0
\end{array}\right.
$$

onde, como faremos daqui por diante, tôdas as somatbrias såo supostas de 1 a $n$.

Estas equações nos conduzem logo às seguintes :

$$
(3,3)\left\{\begin{array}{l}
\sum y_{1}-n A+A 10^{-b c} \sum 10^{-c} x_{1}=0 \\
\sum x_{1} y_{1} \cdot 10^{-c} x_{1}-A \sum x_{1} 10^{-c} x_{1}+A \cdot 10^{-b c} \sum 10^{-2 c x_{1} x_{1} 0} \\
\sum y_{1} 10^{-c} x_{1}-A \sum 10^{-c} x_{1}+A \cdot 10^{-b c} \sum 10^{-2 c} x_{1}=0
\end{array}\right.
$$


202

Anais da E. S. "Luiz de Queiroz"

De $(3,3)$ obtemos pela aplicação do teorema de Rouché a equação :

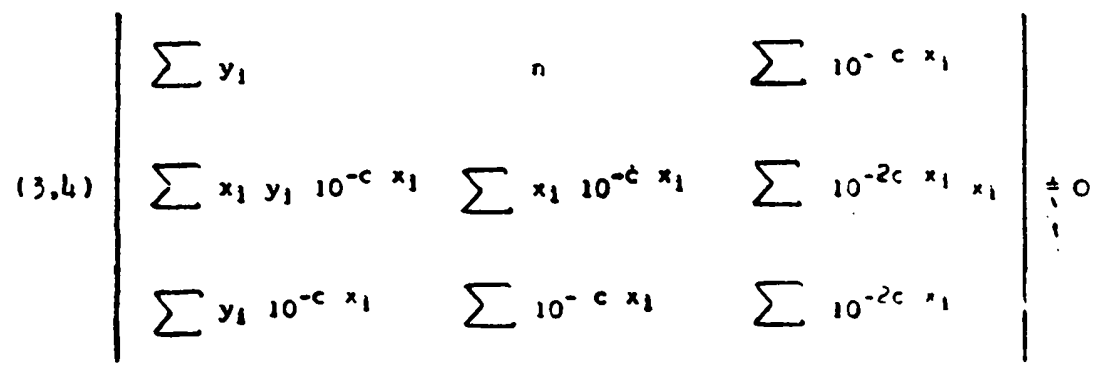

Esta última equação só encerra $c$ como incógnita. Sua resolução pode ser obtida pelos métodos de aproximação da Algebra. O processo é, porém, trabalhoso e demorado. Uma vez calculado $c$, a determinação de $A$ e $b$ a partir das equações $(3,3)$ é simples e cômoda.

Poderíamos, porém, tentar o método dos momentos, também clássico na Estatística. Teríamos então de igualar os momentos de ordem 0,1 e 2 obtidos com os dados observados com os momentos respectivos conseguidos a partir das produções calculadas. Chega-se assim às equações :

$$
\begin{aligned}
& (3.5)\left\{\begin{array}{l}
\sum y_{1}=A \sum\left[1-10^{-c}\left(x_{1}+b\right)\right] \\
\sum x_{1} y_{1}=A \sum x_{1}\left[1-10^{-c} c\left(x_{1}+b\right)\right] \\
\sum x_{1}^{2} y_{1}=A \sum x_{1}^{2}\left[1-10^{-c} c\left(x_{1}+b\right)\right] \\
D(3,5) \text { obtemos : } \\
\sum y_{1}=n A-A \cdot 10^{-b c} \sum 10^{-c} x_{1} \\
\sum x_{1} y_{1}=A \sum x_{1}-A \cdot 10^{-b c} \sum x_{1} 10^{-C} x_{i} \\
\sum x_{1}^{2} y_{1}=A \sum x_{1}^{2}-A \cdot 10^{-b c} \sum x_{1}^{2} 10^{-C} x_{1}
\end{array}\right.
\end{aligned}
$$


Pelo teorema de Rouché, conseguimos a equação seguinte, na qual foram eliminados $A$ e $b$.

$(3.7)\left|\begin{array}{lll}\sum y_{1} & n & \sum 10^{-c \cdot x_{1}} \\ \sum x_{1} y_{1} & \sum x_{1} & \sum x_{1} \cdot 10^{-c} x_{1} \\ \sum x_{1}^{2} y_{1} & \sum x_{1}^{2} & \sum x_{1}^{2} 10^{-c} x_{1}\end{array}\right|=0$

Esta equação é notàvelmente mais simples do que $(3,4)$, pois todos os elementos das duas primeiras colunas são independentes de $c$ e fáceis de calcular com os dados experimentais.

Também neste caso é fácil obter os valores de $A$ e $b$, depois de terminado o cálculo de $c$, com $o$ auxílio das equações $(3,6)$. Aliás das duas primeiras tiramos logo:

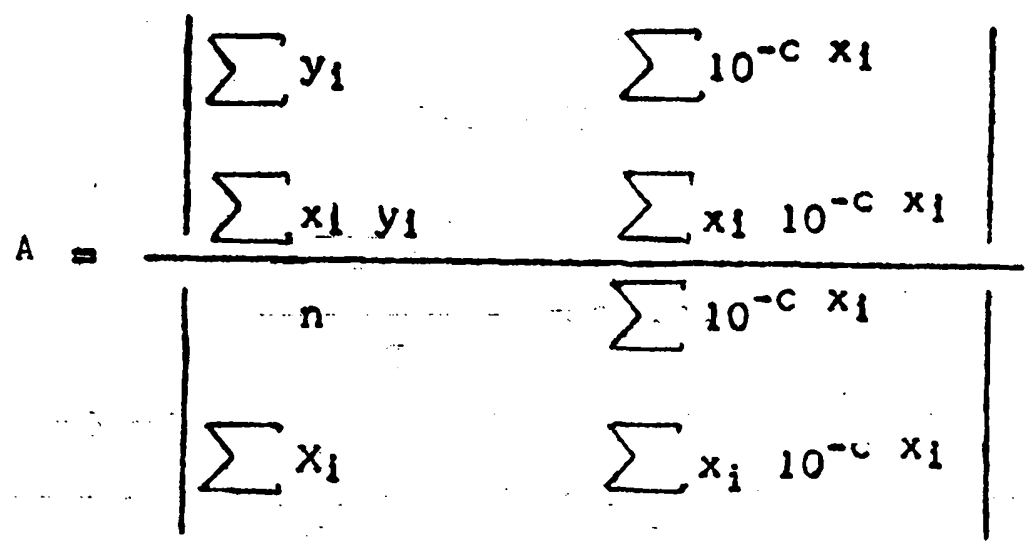

e a primeira nos dá

$$
b=\frac{1}{c} \log \cdot \frac{A \sum 10-c x_{i}}{n A-\Sigma y i}
$$


Vejamos um caso particular bastante frequente.

Fixemos $x i=0, x 2=p, x 3=2 p, \ldots, x n=(n-1) p e$ teremos

$$
\Sigma 10-\mathrm{c} \mathrm{xi}_{\mathrm{i}}=\frac{1-10-\mathrm{n} \mathrm{c} \mathrm{p}}{1-10-\mathrm{c} \mathrm{p}}
$$

Esta fórmula, que se estabelece fàcilmente com o auxílio da teoria das progressões geométricas, nos dá por derivação em relação a - c :

$$
\begin{aligned}
& \Sigma 10-c x_{i_{x_{i}}}=\text { p. } \frac{(\mathrm{n}-1) 10-(\mathrm{n}+1) \mathrm{cp}-\mathrm{n} 10-\mathrm{ncp}+10 \mathrm{cp}}{(1-10-\mathrm{cp}) 2} \\
& \Sigma 10-c x_{i_{x_{i}}} 2=\left[\begin{array}{l}
-(n-1)^{2} 10-\left(n+{ }^{2}\right) c p+\left(2 n^{2}-2 n-1\right) 10-(n+1) c p- \\
-n^{2} 10-n c p+10-2 c p+10-c p
\end{array}\right] \\
& \times \frac{p^{2}}{(1-10-c p) 3}
\end{aligned}
$$

As últimas três fórmulas substituidas em $(3,7)$ nos dão uma equação de grau $n+2 \mathrm{em} 10-\mathrm{cp}$. Sua resolução em geral só será possível por processos numéricos de aproximação. Sendo $k$ uma das raízes teremos :

$$
\begin{gathered}
10-\mathrm{cp}=\mathrm{k}, \\
\therefore \quad \mathrm{c}=\frac{\log \frac{1}{\mathrm{k}}}{\mathrm{p}}
\end{gathered}
$$

Como $p$ é positivo e $c$ também deve ser positivo, é preciso que tenhamos $\log \frac{1}{k}>0, \log \frac{1}{k}>1$ e portanto $0<\mathbf{k}<1$. Raízes que não satisfaçam a esta condição não nos podem convir.

Note o leitor que o que se denomina geralmente na literatura "método dos quadrados mínimos", tendo em vista a aplicação da função de MITSCHERLICH, está longe de ser realmente a aplicação do método dos quadrados mínimos. Com efeito, da função 
obtém-se

$$
y=A[1-10-(x+b)],
$$

$$
z=\log \frac{A}{A-y}=c(x+b) \text {. }
$$

Admite-se então um valor de A próximo das produções máximas observadas. Então para cada valor yi calcula-se o vaA

lor de $z i=\log \frac{A}{A-y i}$. Com os valores de zi e xi obtémse então pelos métodos correntes de interpolação a função

$$
z=c(x+b) .
$$

Para cada valor de A obtém-se uma função dêsse tipo. Escolhe-se a que der uma interpolação mais precisa.

Os autores, como, por exemplo, SARAIVA (13, pp. 17-18), HOOVER e NORMAN (17, p. 333) e REINHOLD (26, p. 883), denominam o método que acabamos de expor de "método dos quadrados mínimos". E' evidente o êrro dessa denominação. $\mathrm{Na}$ realidade os autores não encontraram, em tôda a literatura consultada, nenhuma aplicação do verdadeiro método dos quadrados mínimos. A exposiçẫo que dêsse método fizemos atrás, parece ser a primeira adaptação do referido método ao caso da interpolação com a função de MITSCHERLICH.

\section{4 - UM EXEMPLO DE APLICAÇÃO DO MÉTODO DOS MOMENTOS}

Vejamos agora como se faz numèricamente a determinação dos parâmetros. Utilizaremos para isso os dados publicados por SARAIVA $(13$, p. 25$)$ referentes a uma experiência de adubação com potássio e reproduzidos a seguir. Cada um dos números representativos da produção obtida é média de 5 colheitas. Sob o ponto de vista estatístico, é sempre preferível utilizar na interpolação os dados originais e não médias. No caso vertente, porém, o resultado não é alterado pelo uso das médias. Pois o seu uso neste caso corresponde a dividir por 5 a primeira coluna do determinante de $(3,7)$, o que não altera a equação.

Os dados em que nos baseamos foram os seguintes, referentes à colheita em gramas de aveia semeada em vasos de $20 \mathrm{~cm}$ de diâmetro ( $314 \mathrm{~cm}^{2}$ de área). 
Adubo por vaso ( $\mathrm{K} 2 \mathrm{O}$

$\begin{array}{llllll}0 & 0,2 & 0,3 & 0,5 & 0,8 & 1,5\end{array}$

Colheitas observadas

(Médias de 5 vasos) $\quad 30,0 \quad 72,7 \quad 80,5 \quad 89,6 \quad 94,3 \quad 92,1$

Daí tiramos:

\begin{tabular}{r|r|r|r|r}
\hline$x i$ & $y i$ & $x i$ yi & $x i 2 y i$ & $x i 2$ \\
\hline 0,0 & 30,0 & - & - & - \\
0,2 & 72,7 & 14,54 & 2,908 & 0,04 \\
0,3 & 80,5 & 24,15 & 7,245 & 0,09 \\
0,5 & 89,6 & 44,80 & 22,400 & 0,25 \\
0,8 & 94,3 & 75,44 & 60,352 & 0,64 \\
1,5 & 92,1 & 137,15 & 207,225 & 2,25 \\
\hline 53,3 & 459,2 & 296,08 & 300,130 & 3,27 \\
\hline
\end{tabular}

De acôrdo com $(3,7)$ a equação a resolver será então

$$
\left(4.11\left|\begin{array}{ccc}
459,2 & 6 & \sum 10^{-c} x_{1} \\
296,08 & 3,3 & \sum x_{1} 10^{-c} x_{1} \\
300,13 & 3,27 & \sum x_{1}^{2} 10^{-c} x_{1}
\end{array}\right|=0\right.
$$


Poderíamos iniciar o cálculo tomando como ponto de partida um valor qualquer como, por exemplo, o valor de $c$ determinado por MITSCHERLICH para o caso do potássio. Esse valor é $c=0,93$ quando 'referido a quintais-métricos por hectare. Ora, nós sabemos que

$$
\frac{d y}{d x}=k(A-y),
$$

com $(A-y)$ expresso em quintais por hectare.

Mas no caso vertente $(A-y)$ aparece multiplicado por 314 (área do vaso) e por 100.000, que é o número de gramas em um quintal métrico, e. dividido por 100.000 .000 , que é o número de centímetros quadrados em um hectare. Resulta que teremos

$$
\frac{d y}{d x}=k^{\prime}(A-y) 314 \cdot \frac{10^{5}}{10^{3}}=k^{\prime} \cdot 0,314(A-y)
$$

Logo, devemos ter

$$
\mathbf{k}=\mathbf{k}^{\prime} \mathbf{0 , 3 1 4}
$$

Multiplicando-se por $\mathrm{L} e=0,4343$, vem enfim

$$
c=c^{\prime} \cdot 0,314
$$

Segue-se que temos

$$
c^{\prime}=\frac{0,93}{0,314}=2,96
$$

Em vez de partir dêsse valor nós preferimos, porém, partir do que foi determinado por SARAIVA (13) pelo método dado por KLETSCHKOWSKY e SHELESNOW (14). Esse valor, que foi $c=2,39$, nos deu o cálculo seguinte: 
Anais da E. S. "Luiz de Queiroz"..

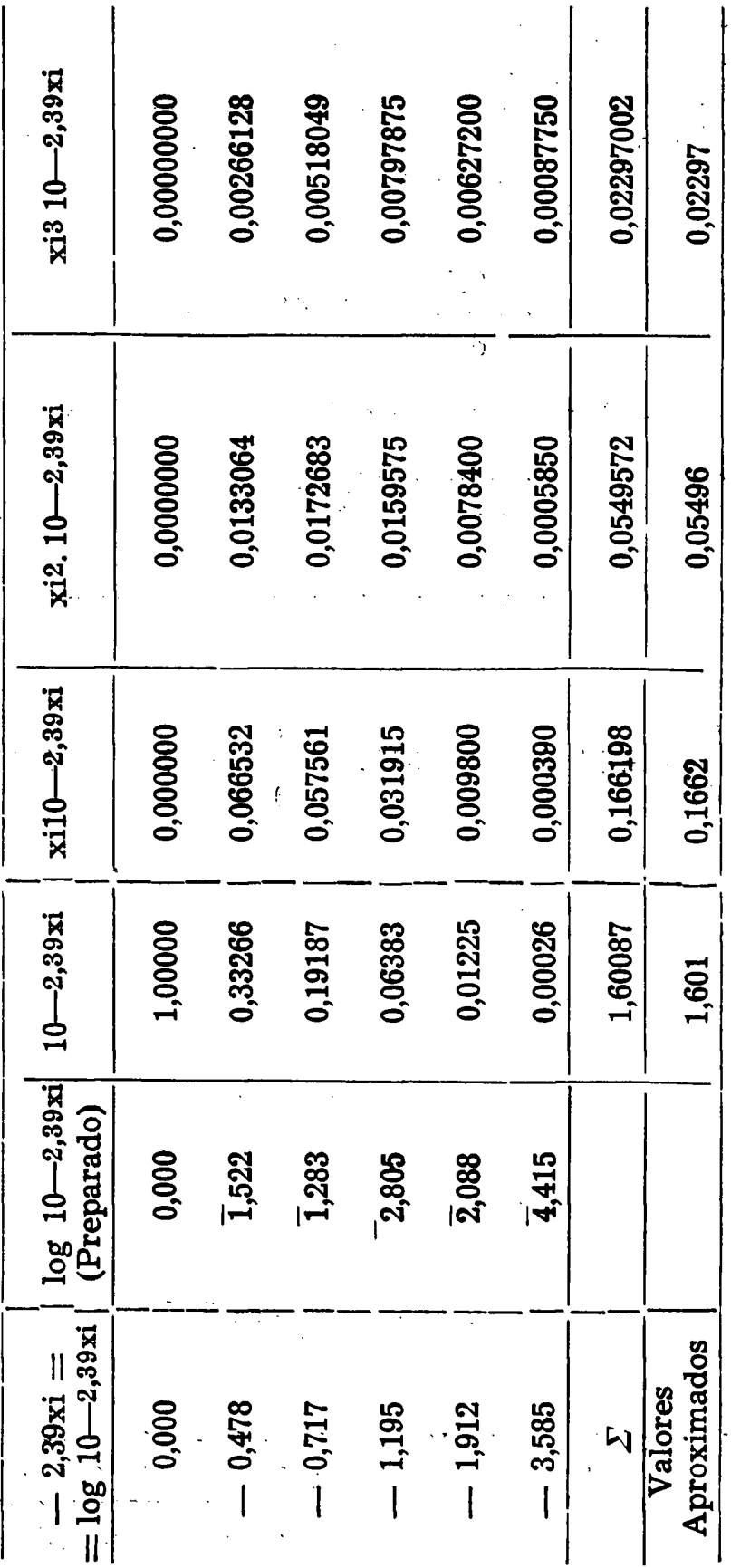


$(4,1)$ nos dá pela regra de SARRUS :

$(4,2) \quad f(c)=-22,247 \Sigma 10-c x i+$ $+299,2 \Sigma x i 10-c x i-261,12 \Sigma x i 210-c x i$

Para $c=2,39$ temos então

$$
\begin{gathered}
\mathrm{f}(\mathrm{c})=-22,247 \times 1,601+299,2 \times 0,1662-261,2 \times 0,05496 \\
=-0,243786 \\
. \quad \mathrm{f}(\mathrm{c}) \sim-0,2438
\end{gathered}
$$

Para conseguir um valor de $c$ mais aproximado calculemos f' (c) . (4,9) nos dá

$f^{\prime}(c)=\left[\begin{array}{c}-22,247 \Sigma x i 10-c x i+299,2 \Sigma x i 210-c x i- \\ -261,12 \Sigma x i 310-c x i\end{array}\right](-L 10)$

Portanto temos

$\left.f^{\prime}(c)=\left[\begin{array}{c}-22,247 \times 0,1662+299,2 \times 10,05496- \\ -261,12 \times 0,02297\end{array}\right]=-2,3\right)=$

Seja $c^{\prime}=c+\triangle c$ o valor que procuramos, tal que $f\left(c^{\prime}\right)=0$. Temos pela fórmula de TAYLOR

$$
\mathrm{f}(\mathrm{c}+\Delta \mathrm{c})=\mathrm{f}(\mathrm{c})+\frac{\Delta \mathrm{c}}{1} \mathrm{f}^{\prime}(\mathrm{c})+\frac{\Delta \mathrm{c}^{2}}{2^{\prime}} \mathrm{f}^{\prime \prime}(\mathrm{c})+\ldots
$$

Se $\Delta \mathrm{c}$ é pequeno podemos desprezar sem grande êrro todos os têrmos do segundo membro a partir do terceiro. $\mathbf{E}$ fica

$$
\begin{gathered}
0=f(c)+\Delta c f^{\prime}(c) \\
\Delta c=-\frac{f(c)}{f^{\prime}(c)}=-\frac{-0,244}{-15,522}=-0,016
\end{gathered}
$$

Logo temos

$$
c^{\prime}=c+\triangle c=2,39-0,016=2,374 \text {. }
$$

Agora calculamos, de maneira análoga à que adotamos antes, o valor de $f(c)$ para $c=2,374$. Achamos $f(c)=0,010380 \sim$ $\sim 0,0104$. Vê-se que êsse valor já é bem aproximado. Mas para obter a aproximação ainda maior sem precisar calcular f' (c) podemos proceder como se segue. 
Quando c varia de 2,374 para 2,39 f (c) varia de 0,01038 para - 0,2438. Logo, se a variação fôsse exatamente proporcional teríamos :

$$
\begin{gathered}
0,25418 \longrightarrow x+0,016 \\
0,01038=\frac{0,01038 \times 0,016}{0,25418} \sim 0,00065
\end{gathered}
$$

Logo um valor mais exato de $c$ será

$$
c=2,374+0,00065=2,37465 \sim 2,375
$$

Para $c=2,375$ temos no nosso caso $\Sigma 10-\mathrm{cxi} \sim 1,6066, \Sigma$ xi $10-\mathrm{xi} \sim 0,16811$,

$$
\Sigma \mathrm{xi2} 10-\mathrm{cxi} \sim 0,055756 \text {. }
$$

Isso nos dá

$$
f(c)=-0,001067 \text {, }
$$

o que mostra que se trata de uma aproximação já muito boa. Agora calculemos $A$ e b.

$$
\begin{gathered}
A=\frac{\left|\begin{array}{ll}
4.59,2 & 1,6066 \\
296,08 & 0,16811
\end{array}\right|}{\left|\begin{array}{cl}
6 & 1,6066 \\
3,3 & 0,16811
\end{array}\right|} \sim 92,8 \\
\mathrm{~b}=\frac{1}{2,375} \log \frac{92,8 \times 1,6066}{6 \times 92,8-459,2} \sim 0,077
\end{gathered}
$$

A equação de MITSCHERLICH será portanto

$$
y=92,8[1-10-2,375(x+0,077)]
$$

Com seu auxílio obtivemos os valores calculados indicados no quadro seguinte, onde figuram também os números obtidos por SARAIVA seguindo u'a marcha diferente da nossa. 


\begin{tabular}{|c|c|c|c|c|c|c|}
\hline $\mathbf{x}$ & 0 & 0,2 & 0,3 & 0,5 & 0,8 & 1,5 \\
\hline $\begin{array}{ll}\text { y } & \text { (Cálculo nosso) } \\
\text { y } & \text { (Calculo de Saraiva) } \\
\text { y (Observado) }\end{array}$ & $\begin{array}{l}31,9 \\
29,9 \\
30,0\end{array}$ & $\begin{array}{l}72,4 \\
72,3 \\
72,7\end{array}$ & $\begin{array}{l}81,0 \\
81,3 \\
80,5\end{array}$ & $\begin{array}{l}88,8 \\
89,4 \\
89,6\end{array}$ & $\begin{array}{l}92,0 \\
92,7 \\
94,3\end{array}$ & $\begin{array}{l}92,8 \\
93,5 \\
92,1\end{array}$ \\
\hline
\end{tabular}

Como se vê, em qualquer dos casos os valores calculados combinam bem com os observados. Entretanto o valor observado correspondente a $x=1,5$ parece indicar que já começou o efeito prejudicial do adubo, que já foi atingida a zona de depressão onde não é mais aplicável a primeira aproximação de MITSCHERLICH. Isso nos levou a excluir êsse valor e a recalcular tudo. Obtivemos então $c=2,19, \mathrm{~A}=95,3, \mathrm{~b}=0,76$. A equação será portanto

$$
\mathrm{y}=95,3[1-10-2,19(0,076+\mathrm{x})],
$$

equação que nos deu os valores calculados apresentados no quadro seguinte :

\begin{tabular}{c|c|c|c|c|c}
\hline $\mathbf{x}$ & 0 & 0,2 & 0,3 & 0,5 & 0,8 \\
\hline $\mathrm{y}$ (Novo cálculo) & 30,3 & 71,6 & 81,0 & 90,1 & 94,1 \\
$\mathrm{y}$ (Observado) & 30,0 & 72,7 & 80,5 & 89,6 & 94,3 \\
\hline
\end{tabular}

\section{5 - AINDA O METODO DOS MOMENTOS}

A equação $(3,7)$ pode ser resolvida por outro processo, frequentemente mais expedito e que nos permite tirar uma importante conclusão teórica.

Uma vez conhecidas as doses crescentes de adubo $\mathrm{x} 1$, $\mathrm{x} 2, \ldots, \mathrm{xn}$ e as produções correspondentes $\mathrm{y} 1, \mathrm{y} 2, \ldots, \mathrm{yn}, \mathrm{po-}$ demos substituir em $(3,7)$ êsses valores nas duas primeiras colunas, desenvolver o determinante em função dos elementos da 3a. coluna e obter uma equação

$$
\begin{aligned}
& \text { A } \Sigma \text { xi2.10-cxi }+ \text { B } \Sigma \text { xi. 10-cxi }+C \Sigma 10-c x i=0, \\
& \text { análoga a }(4,2) .
\end{aligned}
$$


Suponhamos agora que todos os xi $(i=1,2, \ldots, n)$ são múltiplos de uma mesma quantidade $q$ de adubo. Teremos en-

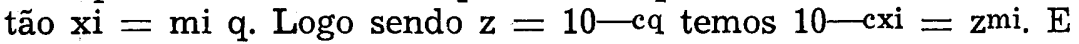
$(5,1)$ nos dá

$$
\begin{array}{cc}
(5,2) & \left(\mathrm{Ax}_{1}^{2}+\mathrm{B} \mathrm{x}_{1}+\mathrm{C}\right) \mathrm{z}^{\mathrm{m} 1}+\left(\mathrm{Ax}_{2}^{2}+\right. \\
\left.+\mathrm{B} \mathrm{x}_{2}+\mathrm{C}\right) & \mathrm{z}^{\mathrm{m} 2}+\ldots+\left(\mathrm{Ax} \mathrm{x}_{\mathrm{n}}^{2}+\mathrm{B} \mathrm{x}_{\mathrm{n}}+\mathrm{C}\right) \mathrm{z}^{\mathrm{mn}}=0
\end{array}
$$

Basta substituir $\mathrm{x} 1, \mathrm{x} 2, \ldots$, xn nesta equação para obter uma equação algébrica em $z$ que se resolverá pelos processos numéricos de aproximação pois, em geral, será de grau elevado. Como $\mathrm{z}=10-\mathrm{cq}$, só interessarão as raízes positivas de $(5,2)$. Mas lá os coeficientes são todos valores de $\mathrm{P}(\mathrm{x})=$ $=A x^{2}+B x+C$ para $\mathrm{x}=\mathrm{x} 1, \mathrm{x} 2, \ldots, \mathrm{xn}$. Sendo $\mathrm{P}(\mathrm{x})$ um trinomio do $2^{\circ}$. grau ao darmos a $x$ valores crescentes êle mudará de sinal no máximo duas vêzes. Logo $(5,2)$ terá no máximo duas raízes positivas pois, como se sabe, o número de raízes positivas de uma equação não pode exceder o número de variações de sinal de seus coeficientes, suposta a equação devidamente ordenada, tal como acontece com a nossa. Mas é fácil verificar que $c=0$ é sempre uma solução para $\cdot(3,7)$. Logo $\mathrm{z}=100=1$ é sempre solução de $(5,2)$, solução que não interessa, porém, pois corresponde a $c=0$. Logo $(5,2)$ nos dará no máximo uma raíz positiva $\mathrm{z}$ diferente de 1 , com a qual calcularemos o valor de $\mathrm{c}$ :

$$
\bar{z}=10-c q, . c=\frac{-\log \bar{z}}{q}=\frac{\log \frac{1}{\bar{z}} .}{q}
$$

Como $c$ deve ser positivo, devemos $\operatorname{ter} \log \frac{1}{\bar{z}}>0$,
$\log \frac{1}{\bar{z}}>1$, e portanto $\quad 0<\bar{z}<1$.

\section{6 - UM MAU EXEMPLO DA LITERATURA}

Para esclarecer melhor o que expuzemos no capítulo anterior e também para mostrar os perigos que podem advir da aplicação indiscriminada da teoria de MITSCHERLICH veremos um exemplo fornecido por JORET (12, p. 180). Esse au- 
tor fez experiências de adubação de trigo com nitrogênio e obteve os seguintes dados:

DADOS EM QUINTAIS-METRICOS POR HECTARE

\begin{tabular}{l|l|l|l|l}
\hline Doses de nitrogênio (xi) & 0 & 0,45 & 0,60 & 0,75 \\
\hline Produção de trigo obtida (yi) & 39,4 & 47,4 & 51,2 & 55,4 \\
\hline
\end{tabular}

A produção da testemunha é média de 15 parcelas. As demais são médias de vinte parcelas. Em todos os casos - diz JORET - foi pequeno o êrro experimental.

Com os dados de JORET obtivemos :

$$
\begin{aligned}
\Sigma \text { yi }=193,4, \Sigma & \text { xiyi }=93,600, \Sigma \text { xi2yi }=59,1930, \\
n=4, \Sigma & \text { xi }=1,80, \quad \Sigma \text { xi2 }=1,1250
\end{aligned}
$$

Fomos obrigados a usar as médias por êle apresentadas, pois não dá os dados originais. Aliás, seguindo a errônea praxe repetida em tôda a bibliografia do assunto, JORET também fez seus cálculos tomando por base as médias que reproduzimos acima.

De $(3,7)$ obtemos então a equação

$$
\begin{aligned}
& \left|\begin{array}{lll}
193.4 & 4 & \sum 0^{-c x_{1}} \\
93.6 & 1,8 & \sum x_{1}{ }^{10^{-c x_{1}}} \\
59.193 & 1.125 & \sum x_{1}{ }^{2} 10^{-c x_{1}}
\end{array}\right|=0 \\
& \text { da qual resulta } \\
& -1,242 \sum 10^{-c x_{1}}+19,185 \sum x_{1} 10^{-c x_{1}}-26,28 \sum x_{1}^{2} 10^{-c x_{1}}=0 \\
& \text { Se ja agora } q=0,15 \& 2=10^{-0.15 \mathrm{C}} \text {. obtemos } \\
& -1.242\left(1+2^{3}+2^{4}+2^{5}\right)+19.185\left(0.452^{3}+0.6 z^{4}+0.752^{5}\right) \cdot \\
& -26,28\left(0,20252^{3}+0,36 z^{4}+0,56252^{5}\right)=0 \text {. }
\end{aligned}
$$

ou ainde

$$
I(z)=-1,242+2,069 z^{3}+0,508 z^{4}-1,636 z^{3}=0 .
$$


Sabemos que uma raíz é $z=1$. A outra raiz positiva verificámos ser, aproximadamente, $\vec{z}=1,17$. Ora, nós sabemos que $z$ deveria ser menor que 1 . Isto indica logo uma anomalia. $O$ valor de $c$ correspondente é

$$
c=\frac{-\log 1,17}{0,15}=-0,4546,
$$

o que representa um absurdo, pois $c$ deve ser positivo. $O$ valor de A correspondente, calculado com auxílio de $(3,8)$ é 25,92, o que também é evidentemente um absurdo, pois tal "produção máxima" é menor que qualquer das produçōes obtidas.

Note-se que, com auxílio do método mais simples discutido por nós em trabalho anterior (2) obtivemos com os dados de JORET $\mathrm{c}=-0,455$ e $\mathrm{A}=26,05$, valores que concordam muito bem com os obtidos pelo método dos momentos.

No entanto JORET determinou com êsses mesmos dados valores de $c$ todos positivos e tendo como média 0,329 .

Qual a marcha seguida por JORET ?

JORET considerou as equações

$$
\begin{aligned}
& \log (A-y 1)=\log A-c(x 1+b), \\
& \log (A-y 2)=\log A-c(x 2+b), \\
& \log (A-y 3)=\log A-c(x 3+b), \\
& \log (A-y 4)=\log A-c(x 4+b),
\end{aligned}
$$

em que os valores de $x$ e $y$ são os que figuram no quadro visto acima. Combinando três a três essas equaçōes, JORET obteve 4 sistemas distintos de três equaçöes a três incógnitas e a partir de cada um deles determinou os valores de $A, b, c$, por processo numérico que não explica bem qual foi. Os valores por êle obtidos por êsse método foram os seguintes :

$\begin{array}{llll}\text { Com x1, x2, x3 } & 62,5 & 1,08 & 0,409 \\ \text { Com x1, x2, x4 } & 67,0 & 1,15 & 0,330 \\ \text { Com x1, x3, x4 } & 72,7 & 1,06 & 0,319 \\ \text { Com x2, x3, x4 } & 91,2 & 0,76 & 0,261\end{array}$

Em nosso trabalho anterior (2) discutimos detalhadamente a resolução, por métodos algébricos, de sistemas de equações como os utilizados por JORET. O método que lá vimos exige 
que os valores de $x$ estejam em progressão aritmética e portanto só pode ser usado para o caso de tomarmos os dados referentes a x2, x3, x4. Obtemos então

$$
\begin{aligned}
& \log (A-y 2)=\log A-c(0,45+b) \\
& \log (A-y 3)=\log A-c(0,60+b) \\
& \log (A-y 4)=\log A-c(0,75+b),
\end{aligned}
$$

e daí se obtém seguindo a marcha já por nós examinada (2) :

$$
A=-\frac{y_{3}^{2}-y_{4} y_{2}}{2 y_{3}-\left(y_{4}+y_{3}\right)}=\frac{(51,2) 2-47,4 \times 55,4}{2 \times 51,2-(47,4+55,4)}=11,3
$$

O valor de $c$ é $-0,290$.

Tais resultados são evidentemente absurdos. Aliás, êles já se deviam esperar pois, como demonstramos antes (2) para que se aplique a lei de MITSCHERLICH, devemos ter

o que não acontece nesse caso.

$$
2 \mathrm{y}_{3}-\left(\mathrm{y}_{2}+\mathrm{y}_{4}\right)>0,
$$

A importância das experiências de JORET está no fato de que DEMOLON (16, pp. 269-270) as tomou como fundamentais nas suas considerações sôbre o método de MITSCHERLICH.

\section{7 - A TECNICA DAS EXPERIENCIAS EM VASOS}

Tudo o que dissemos até aqui se aplica a experiências tanto em vasos como no campo. A experimentação em vasos apresenta, porém, certas particularidades que discutiremos agora.

Com', a execução do método de MITSCHERLICH em vasos podemos controlar a ação de certos fatores cuja flutuação iria complicar o ensaio no campo. Assim é que conseguimos homogeneizar o meio e regularizar o importante fator água; trabalhando com um número certo de plantas por unidade de área fàcilmente protegêmo-las contra pragas, moléstias e intempéries.

Apesar do artificialismo relativo dessas condições tão cuidadosamente reguladas, a experiência em vasos, segundo DEMOLON (16, pp. 102-103), representa, em vista do que ficou escrito, um método auxiliar capaz de esclarecer o mecanismo de certos fenômenos, o modo de ação de vários fatores da pro- 
dução, o valor cultural comparado dos adubos, servindo assim de guia à experimentação em pleno campo.

Nas experiências em questão usam-se os chamados vasos de MITSCHERLICH, cilíndricos, feitos de ferro esmaltado, brancos por fora e tendo $20 \mathrm{~cm}$ de diâmetro por $20 \mathrm{~cm}$ de profundidade; apresentam na base um orifício de drenagem com $8 \mathrm{~cm}$ de diâmetro, o qual é provido de uma calota metálica que o cobre quando em uso; os vasos descansam sôbre coletores deslocáveis de $24 \mathrm{~cm}$ de diâmetro por $7 \mathrm{~cm}$ de profundidade, coletores êsses que recolhem as águas de drenagem. Para assegurar a posição ereta das plantas e protegê-las contra o vento, usam-se suportes de arames grosso galvanizado, mantidos verticalmente, graças a três aneis do mesmo material. $O$ anel inferior se adapta sôbre o vaso e os outros são um pouco menores, de modo que os suportes podem ser colocados uns dentro dos outros.

Cada vaso é cheio com um quilo de terra tirada de uma profundidade de 0 a $20 \mathrm{~cm}$; passa-se a terra por peneira de $1 / 4$ de polegada de malha e mistura-se (ou não) com cinco quilos de areia lavada : tal diluição é feita só para a terra dos vasos onde se verificará a ação do fósforo e do potássio e tem por fim salientar a reação dêsses fertilizantes. Entretanto para solos fracos a diluição deve ser menor, como por exemplo, de uma parte de terra para duas de areia (perfazendo seis quilos) ou mesmo poderá ser dispensada em se tratando de solos de extrema pobreza.

O ensaio é feito com 4 repetições, usando-se ao todo 20 vasos como segue :

a) Ação do nitrogênio 4 vasos com 6 quilos de solo 4 vasos com 6 quilos de solo

\author{
adubação \\ P K (sem N) \\ P K N (completo)
}

b) Ação do fósforo e potássio

4 vasos com $\left\{\begin{array}{l}1 \mathrm{~kg} \text {. de terra } \\ 5 \mathrm{~kg} \text {. de areia }\end{array} \mathrm{K} \mathrm{N} \quad(\right.$ sem $\mathrm{P})$

4 vasos com $\left\{\begin{array}{l}1 \mathrm{~kg} \text {. de terra } \\ 5 \mathrm{~kg} \text {. de areia }\end{array}\right.$ P N (sem K)

4 vasos com $\left\{\begin{array}{l}1 \mathrm{~kg} . \text { de terra } \\ 5 \mathrm{~kg} \text {. de areia }\end{array}\right.$ P K N (completo) 
Os diversos adubos são adicionados da seguinte maneira:

a) $1,1 \mathrm{~g}$ de $\mathrm{N}$ como $\mathrm{NH} 4 \mathrm{NO} 3$ em $20 \mathrm{~cm} 3$ de solução por vaso

b) $1,5 \mathrm{~g}$ de $\mathrm{K} 2 \mathrm{O}$ como $\mathrm{K} 2 \mathrm{SO} 4 \mathrm{em} 50 \mathrm{~cm} 3$ de solução por vaso

c) 1,0 g de P2O5 como Ca (H2PO4) 2. $\mathrm{H} 20$ em $50 \mathrm{~cm} 3$ de solução por vaso

d) 0,5 g de $\mathrm{NaCl}+0,5 \mathrm{~g}$ de $\mathrm{MgSO} 4$ em $5 \mathrm{~cm} 3$ de solução por vaso

e) $1,5 \mathrm{~g}$ de $\mathrm{CaCO} 3$ por vaso.

O $\mathrm{CaCO}$, que é o único adubo adicionado em estado sólido incorpora-se a todos os vasos; MITSCHERLICH não o usava, embora o recomendasse, porque a água de canalização do Instituto Agronômico de Königsberg é rica de cal. $\mathrm{O} \mathrm{NaCl}$ é adicionado a todos os vasos; tem por fim facilitar o aproveitamento do $\mathrm{K} 2 \mathrm{O}$, que de outro modo atuaria dificientemente. $\mathrm{O}$ MgSO4, também incorporado a todos os vasos, tem o objetivo de garantir culturas convenientes. tadas.

A mistura da terra com os adubos faz-se em bacias. esmal-

A adição do $\mathrm{CaCO} 3$ é feita em primeiro lugar. A seguir juntam-se as soluções apropriadas com auxílio duma pipeta, cujo jato é dirigido de modo a tornar a distribuição o mais uniforme possível. Então mistura-se tudo muito bem e transferese o solo adubado para o vaso respectivo, através de um grande funil de ferro esmaltado.

Comprime-se com as mãos a primeira camada $(5 \mathrm{~cm}$ mais ou menos) de solo posta no vaso; o restante poderá ficar mais sôlto. Arranjam-se os vasos de tal modo que aqueles igualmente adubados não permaneçam adjacentes e muda-se a situação das diversas séries durante a experiência de modo a eliminar efeitos de sombra.

As sementes de aveia (Avena sativa L.) - que é a planta-reativo (-) escolhida por MITSCHERLICH - deverão ter sido anteriormente desinfetadas com Uspulam ou outro fungicida apropriado. Usa-se uma tábua de pequena espessura e provida de cinco filas de orifícios de $0,5 \mathrm{~cm}$ de diâmetro e equidistantes para praticar no solo previamente umidecido 25 covetas de $0,5 \mathrm{~cm}$ de diâmetro por $1,5 \mathrm{~cm}$ de profundidade e afastadas entre si $3 \mathrm{~cm}$. Cada buraco recebe duas sementes com o embrião voltado para baixo e a seguir é fechado cui-

(-) Para paises tropicais MITSCHERLICH recomenda, em lugar de aveia, arroz (Oriza sativa L.) ou sôrgo (Andropogon sorghum) 
dadosamente. Enquanto os "seedlings" não emergem, umidece-se a terra ligeiramente uma ou duas vêzes por dia, mantendo-se os vasos cobertos com esteiras de fôlhas (13, pág. 8) ou com os coletores (18).

O desbaste é feito 14 dias após a semeadura, deixando-se 35 plantas por vaso.

Verificada a germinação, regula-se o fator água como segue : na primeira semana os vasos deverão conservar $50 \%$ do poder de embebição da mistura terra mais areia, o que se consegue mediante pesagem diária e restituição de água evaporada; na 2a. semana, $80 \%$, na $3 a$. $95 \%$, na $4 a$. semana e seguintes, junta-se água até completa embebição, isto é, até começar a gotejar no coletor; esta água assim recolhida, deverá ser restituida ao vaso respectivo no dia seguinte fazendo-se depois a irrigação p. d. Aproximando-se a maturação diminui-se a quantidade de água, suspendendo-se totalmente as regas logo que os grãos deixem de "dar leite" (13, pág. 8) (8 dias antes da colheita, aproximadamente).

A colheita é feita como no método de HELLRIEGEL : cortam-se, com tesoura, as hastes ao nível do colo. Palha e grãos depois de sêcos ao ar são pesados separadamente. Somando-se o pêso da palha e o dos grãos sêcos tem-se a colheita total.

\section{8 - A INTERPRETAÇÃO DOS RESULTADOS}

A colheita média obtida nos vasos que receberam $\mathrm{N} P \mathrm{~K}$ representa o máximo que se pode obter mediante adição dêsses três elementos nas condições do ensaio. As colheitas médias com tratamentos sem N, sem P2O5 e sem K2O são calculados como percentagens da colheita máxima. Como exemplo usaremos os seguintes dados colhidos numa experiência de MELLO MORAES e COURY (1, pp. 450-451) :

Tratamento Vasos cheios com

$\begin{array}{cc}N \text { P K } & \text { terra e areia } \\ N \text { K } & \text { terra e areia } \\ N \text { P } & \text { terra e areia } \\ N \text { P K } & \text { terra } \\ \text { P K } & \text { terra }\end{array}$

Colheita média \% do máximo (grãos)

$\begin{array}{rlr}66,75 & \mathrm{~g} & 100 \\ 5,730 & \mathrm{~g} & 8,583 \\ 42,375 & \mathrm{~g} & 63,393 \\ 72,165 & \mathrm{~g} & 100 \\ 9,962 & \mathrm{~g} & 13,800\end{array}$


Para o fósforo tem-se $c=0,60$ e para o potássio em presença de sódio, $c=0,93$. Consultando a tabela transcrita em nosso trabalho anterior (2) e interpolando, verificamos que a quantidade de $\mathrm{P} 2 \mathrm{O} 5$ que possibilita uma colheita igual a $8,583 \%$ da colheita máxima é 0,06486 quintais por hectare; para o K2O achamos 0,46991 quintais por hectare. Com auxílio da tabela dada por DEMOLON (16, p. 277) e interpolando, verificamos que a quantidade de $\mathrm{N}$ correspondente a $13,8 \%$ é de 0,52916 quintais por hectare. Como na experiência a terra foi diluida a 1/6 e considerando que, segundo MITSCHERLICH, a planta extrai metade dos elementos nutritivos dos $20 \mathrm{~cm}$ superficiais e outra metade dos $20 \mathrm{~cm}$ logo abaixo, segue-se que as quantidades de elementos fertilizantes realmente existentes na terra do ensaio são:

$$
\begin{aligned}
& 6 \times 2 \times 0,06486=0,778 \text { quintais de } \mathrm{P} 2 \mathrm{O} 5 / \mathrm{ha} \\
& 6 \times 2 \times 0,46991=5,638 \text { quintais de } \mathrm{K} 2 \mathrm{O} / \mathrm{ha} \\
& 2 \times 0,52916=1,058 \text { quintais de } \mathrm{N} / \mathrm{ha}
\end{aligned}
$$

Voltando às tabelas verificamos que os 0,778 quintais de P2O5 por hectare garantem $65,5 \%$ da colheita máxima. A quantidade de $\mathrm{K} 2 \mathrm{O}$ é tão grande que não figura na tabela e, por conseguinte, facultará $100 \%$ do máximo. Finalmente, 1,058 quintais de $\mathrm{N} /$ ha asseguram $25,718 \%$ do máximo de produção possível. Em conclusão : o terreno analisado necessita de uma adubação fosfatada de $2,20-0,778=1,422$ quintais de $\mathrm{P} 2 \mathrm{O} 5$ por ha (-) dispensando adubação potássica. A necessidade de adubos nitrogenados é evidente: entretanto não trataremos dêste ponto devido às contraindicações existentes a respeito da aplicação do método de MITSCHERLICH para o nitrogênio (10).

\section{9 - COMENTÁRIOS SOBRE AS EXPERIÊNCIAS EM VASOS}

Uma das exigências que nem sempre se compreendem bem na técnica de experiências em vasos aconselhada por MITSCHER-

(-) 2,20 Qt de P205 por ha facultam 95,2\% da colheita máxima; admite-se êsse rendimento como muito vantajoso econômicamente não sendo interessante a incorporação de quantidades maiores de P205 pois os acréscimos nas colheitas seriam muito pequenos ("law of the diminishing returns"). 
LICH é a diluição da terra em estudo com areia. Procuremos demonstrar objetivamente a vantagem e até a necessidade dessa prática.

Admitida a função de MITSCHERLICH

$$
\mathrm{y}=\mathrm{A}[1-10-\mathrm{c}(\mathrm{x}+\mathrm{b})],
$$

num vaso sem o adubo em questão temos $x=0$ e nos vasos adubados $\mathrm{x}=\mathrm{x} 1$.

Obtemos assim

$$
\begin{gathered}
\text { yo }=A[1-10-\mathrm{cb}] \\
\mathrm{y} 1=\mathrm{A}[1-10-\mathrm{c}(\mathrm{x} 1+\mathrm{b})]
\end{gathered}
$$

Logo temos
$(9,2)$
$\mathrm{d}=\mathrm{y} 1-$ yo $=$ A. $10-\mathrm{bc}[1-10-\mathrm{cx} 1]$.

E' preciso, porém, considerar o seguinte:

1 - A equação $(9,1)$ só se aplica bem para doses não muito grandes de fertilizantes, isto é, para valores de $b$ e $x$ relativamente pequenos. Se o solo for muito rico, a função interpoladora será pouco apropriada e poderá, portanto, conduzir a resultados errôneos.

2 - A diferença y1 - yo diminui à medida que cresce $b$, tendendo para zero quando $b \longrightarrow \cdots$. Logo, para solos relativamente ricos essa diferença, que seria pequena, pode fàcilmente anular-se, e até tornar-se negativa devido à variação inerente a todo trabalho experimental.

Diluindo-se a terra com areia, na proporção de uma parte de terra para 6 de mistura, o valor de $b$ passa a ser $\frac{b}{6}$ e por-
tanto vamos ter

$$
d_{1}=\bar{y}_{1}-\bar{y}_{0}=A 10^{-\frac{c b}{b}}\left[1-10^{-c x_{1}}\right]
$$

\section{Vê-se logo que}

$$
\frac{d_{1}}{d}=10^{\frac{5}{6} b c}, \therefore d_{1}=10^{\frac{5}{6} b c} d .
$$


Logo, se tivermos, como na experiência que vimos atrás, $\mathrm{b}=5,638$ quintais de $\mathrm{K} 2 \mathrm{O}$ e se tomarmos $\mathrm{c}=0,93$, como faz MITSCHERLICH, teremos

$$
\mathrm{d} 1=10^{\frac{5}{6} \cdot 5,638.0,93} \mathrm{~d}=23413 \mathrm{~d},
$$

isto é, a nova diferença multiplicada por 23413 ! E como utilizaremos um meio mais pobre, a função de MITSCHERLICH nos dará uma interpolação mais precisa, mais digna de confiança.

Quanto a quantidade ótima de fertilizante, MITSCHERLICH admitia que seria tal que proporcionasse uma colheita igual a 95\% de A. Essa porcentagem é, porém, evidentemente arbitrária e deve variar com o preço da colheita obtida. Em trabalho anterior (2) os autores propuzeram que a quantidade $x$ ideal de fertilizante a ser acrescentado ao solo fosse dada pela equação

$$
\log \frac{\mathrm{ft} \log \mathrm{e}}{\mathrm{s} \mathrm{c}}=\log \mathrm{A}-\mathrm{c}(\mathrm{x}+\mathrm{b}),
$$

onde, além das letras usuais, temos $t$ indicando o preço de um quintal-métrico de fertilizante, $s$ representando o custo de igual quantidade da produção obtida e $f$, que é uma constante que terá um valor maior que um a ser determinado experimentalmente.

\section{0 - CONCLUSOEES}

I - A interpolação com a lei de MITSCHERLICH

$$
y=A[1-10-c(x+b)]
$$

pode ser feita pela teoria dos quadrados mínimos, mas é conseguida com maior facilidade pelo método dos momentos.

II - Ao fazer a interpolação é preciso que o operador se assegure da possibilidade da aplicação criteriosa da lei de MITSCHERLICH ao caso em estudo. 
III - Os métodos aproximados de interpolação, principalmente se utilizados sem devidos cuidados, podem conduzir a resultados absolutamente ilusórios.

IV - Entre os métodos de uso condenável incluimos o que se utiliza da equação

$$
z=\log \frac{A}{A-y}=c(x+b),
$$

calculando $z$ com um valor mais ou menos arbitrário dado a $\mathbf{A}$ e obtendo a seguir $c$ e $b$ pelos métodos usuais de correlação. Tal método supõe implìcitamente a possibilidade de uma estimativa prévia de $\mathrm{A}$, possibilidade que só existe nos casos em que a lei de MITSCHERLICH se aplica com precisão. $O$ exemplo de JORET nos mostra claramente que isso nem sempre se dá e que a escolha mais ou menos arbitrária de uma estimativa para $A$ pode conduzir a resultados inteiramente ilusórios. Com efeito no referido exemplo o operador seria conduzido a tomar para A um valor próximo da produção máxima obtida $(55,4)$ e aplicaria inocentemente a lei de MITSCHERLICH a êsse caso em que ela não é aplicável. $O$ cálculo algébrico, porém, pelos métodos por nós indicados nos mostra imediatamente a impropriedade do uso da lei nesse caso.

V-A diluição da terra com areia é aconselhável nas experiências em vasos, afim de permitir a aplicação segura da lei de MITSCHERLICH.

Nas experiências de campo, onde isso não é possível, a aplicação dessa lei pode ser, portanto, indevida. Nelas será imprescindível a verificação, pelo método dos momentos ou pelo método dos quadrados mínimos, de que é lícita a aplicação da lei de MITSCHERLICH. Quanto mais rico fôr o solo, mais suspeita será a sua aplicação a experiência de campo.

VI - O exemplo de JORET nos mostra a necessidade de uma crítica judiciosa de tôda a bibliografia sôbre o assunto, bibliografia essa quase tôda fundada em métodos grosseiros de interpolação, em cálculos apenas baseados em médias, em dados cuja interpretação estatística é falha ou ausente.

VII - O método de MITSCHERLICH será de valor, sem dúvida, como método auxiliar no estudo da necessidade de adubação dos nossos solos. Para aplicá-lo com critério entre nós torna-se necessário, porém, estabelecer uma sólida base experimental para sua utilização. 


\section{1 - ABSTRACT}

This paper deals with some mathematical and statistical problems related to MITSCHERLICH's theory of plant growth.

When an experiment is made data are collected which give the amount (xi) of fertilizer used and the corresponding yield (yi) of the crop. What is required is to get a function $\mathrm{y}=$ $=\mathrm{f}(\mathrm{x})$ that interpolates the data obtained. That function must :

1 - Fit reasonably well any similar experiment with any cultivated plant.

2 - Have only a few parameters easy to compute with the experimental data.

RIPPEL and MEYER (3) tried to use FOURIER series and also the series of LEGENDRE polynomials as interpolation functions. FROHLICH (7) and PFEIFFER (8) tried polynomials. But all such functions are arbitrary. It is only with theoretical considerations that such arbitrariness may be avoided. That is why MITSCHERLICH's formula is generally thought to be much better than other interpolation functions. MITSCHERLICH started from the differential equation

$$
\frac{d y}{d x}=k(A-y),
$$

where $k$ and $A$ are positive constants, $x$ is the amount of fertilizer used and $y$ is the yield produced. The solution of that equation, obtained by integration, is

$$
y=A[1-10-c(x+b)]
$$

where $A$ is the maximum producion obtainable with the fertilizer in study, $c=k \cdot \log e$, where $e$ is the basis of the NAPIE- 
RIAN system of logarithms, and $b$ is the amount of fertilizer available in the soil. So MITSCHERLICH's interpolation function depends only on three parameters. Besides that it was shown by numerous experiments that that function fits very well any data obtained with any cultivated plant except when excessively. large amounts of fertilizers are used. For the last case MITSCHERLICH got a second aproach formula

$$
y=A[1-10-(x+b)] 10-s \times 2,
$$

where we have a fourth parameter $s$ called "factor of injury". The corresponding differential equation is

$$
\frac{\log e}{y} \cdot \frac{d y}{d x}=c \frac{10-c(x+b)}{1-10-c(x+b)}-2 s x .
$$

We must note here that RUSSEL $(10$, p. 140$)$ says that the corresponding differencial equation is

$$
\frac{1}{y} \cdot \frac{d y}{d x}=c \frac{A-y}{y}-2 s x .
$$

It is easy to see that this is not right and, by reading one of MITSCHERLICH's articles $(11$, p. 276), to discover that the source of error was a careless study of that article.

The authers showed in another paper (2) a good method for fitting MITSCHERLICH's first approach formula, a method based on the differential equation

$$
\frac{\mathrm{dy}}{\mathrm{dx}}=\mathrm{k}(\mathrm{A}-\mathrm{y})
$$

and already used, with a few minor changes, by HANKINS (27).

In this paper the authors discuss the fitting of MITSCHERLICH's firtst approach formula with the aid of the method of least squares and PEARSON's method of moments. By the firtst method we get the equations 
Aspectos matemáticos e estatísticos

225

$$
(10.4)\left\{\begin{array}{l}
\sum_{1=1}^{n} y_{1}-n A+A 0^{10-D C} \sum_{1=1}^{n} 10^{-c x_{1}}=0 \\
\sum_{i=1}^{n} x_{1} y_{1} 10^{-c x_{1}}-A \sum_{i=1}^{n} x_{1} 10^{-c x_{1}}+A \cdot 10^{-b c} \sum_{i=1}^{n} 10^{-2 c x_{i}} x_{1}=0 \\
\sum_{1=1}^{n} y_{1} 10^{-c x_{1}}-A \sum_{i=1}^{n} 10^{-c x_{1}}+A, 10^{-b c} \sum_{i=1}^{n} 10^{-2 s x_{1}}=0
\end{array}\right.
$$

From these equations we get by ROUCHE's theorem

$$
110.5)\left|\begin{array}{lll}
\sum y_{1} & n & \sum{ }_{10^{-c x_{1}}} \\
\sum x_{1} y_{1} 10^{-c x_{1}} & \sum_{x_{1} 10^{-c x_{1}}} & \sum \sum_{10^{-2 c x_{1}}} \\
\sum y_{1} 10^{-c x_{1}} & \sum 10^{-c x_{1}} & \sum 10^{-2 c x_{1}}
\end{array}\right|=0 .
$$

Generally this equation in c can be solved only by methous of numerical approximation. When $c$ is obtained it is easy to compute $A$ and $b$ with the aid of equations (10.4).

With the use of the method of moments we obtain the equaltrons

$$
\begin{aligned}
& (10.6)\left\{\begin{array}{l}
\sum y_{1}=n A-A \cdot 10^{-b c} \sum 10^{c x_{1}} \\
\sum x_{1} y_{1}=A \sum x_{1}-A \cdot 10^{-b c} \sum x_{1} 10^{-c x_{1}} \\
\sum x_{i}^{2} y_{1}=A \sum x_{i}^{2}-A \cdot 10^{-b c} \sum x_{i}^{2} 10^{-c x_{1}}
\end{array}\right. \\
& \text { from which we get } \\
& (10.7)\left|\begin{array}{lll}
\sum y_{1} & 1 & \sum 10^{-c x_{1}} \\
\sum x_{1} y_{1} & \sum x_{1} & \sum x_{1} 10^{-c x_{1}} \\
\sum x_{1}^{2} y_{1} & \sum x_{1}^{2} & \sum x_{1}{ }^{2} 10^{-c x_{1}}
\end{array}\right|
\end{aligned}
$$


Equation (10.7) is much simpler than (10.5). From it we compute $c$. The authors prove that besides $c=0$ there is only one other real value of $c$ satisfying (10.7). When this value is obtained it is easy to compute

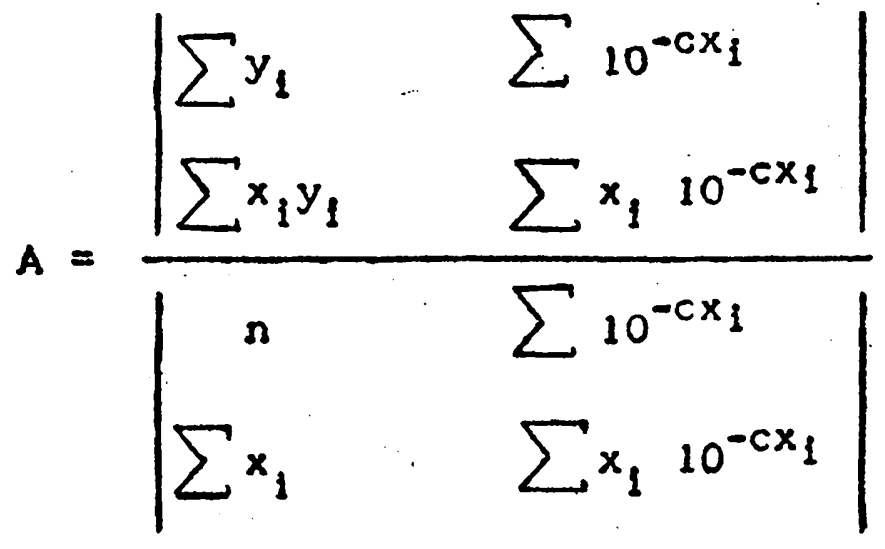

$$
\begin{aligned}
& b=\frac{1}{c} \log \frac{\dot{A} \sum_{n A}-\sum 0^{-c x_{i}}}{\sum y_{i}}
\end{aligned}
$$

The authors show that the so-called "method of least squares" as applied to the interpolation of MITSCHERLICH's first approach formula is not the true method of least squares. It is probable that the authors' is the first exposition of the true method of least squares applied to the interpolation of MITSCHERLICH's function.

Afterwards the authors criticize JORET's (15) interpretation of experimental data obtained by him in France. They show by several methods of computation that MITSCHERLICH's function cannot be applied to JORET's data. The method of moments applied to them gives $c=-0,4546$, that is, a negative value, and $A=25,92$, that is a "maximum production" less than any observed yield. The authors could not explain how JORET, with his data, could obtain a positive mean value 0,329 for $c$ because JORET only says that his computations were made by numerical methods of approximation. JO- 
RET's mistakes are important because DEMOLON (16) took his experiments and computations as fundamental ones in his discussion on MITSCHERLICH's theory.

The authors discuss too the MITSCHERLICH's pot experiment method, showing how data thus obtained are interpreted. They criticize MITSCHERLICH's rule of using as optimum of fertilizer the amount sufficient to produce a yield equal to $95 \%$ of $\mathrm{A}$. Of course the optimum amount of fertilizer for a given soil and crop must change with the price of manures and the price of the yield produced. They remember that in another paper (2) they presented a formula for computing the optimum amount $x$ of fertilizer to be used. That formula is

$$
\log \frac{\text { ft. } 0.4343}{\text { s c }}=\log A-c(x+b),
$$

where $t$ is the price of one hundred kilograms of fertilizer, $s$ is the price of an equal amount of the crop yield and $f$ is a constant greater than one to be experimentally fixed.

\section{2 - BIBLIOGRAFIA CONSULTADA}

1 - MELLO MORAES, J. e Tufi COURY - Refertilização dos solos. Anais do 1o. Congresso Brasileiro de Agronomia, vol. 1, pp. 435-453. Piracicaba. 1940.

2-PIMENTEL GOMES, Frederico e Eurípedes MALAVOLTA - Considerações Matemáticas sôbre a Lei de Mitscherlich. Piracicaba. 1949.

3-RIPPEL, A. e R. MEYER - Ertragsgesetz gegen Wirkungsgesetz. Zeitschrift fur Pflanzenernährung, Düngung und Bodenkunde, pp. 1-24, vol. XIV, parte científica. Berlim. 1929.

4-REDDICK, H. W. e F. H. MILLER - Advanced Mathematics for Engineers. John Wiley \& Sons. Nova York. 1938.

5-FISHER, R. A. - Statistical Methods for Research Workers. Oliver and Boyd. Londres. 1932.

6 - RODRIGUES, Milton da Silva - Elementos de Estatística Geral. S. Paulo. 1934.

7-FROHLICH, Otto - Utber die von E. A. Mitscherlich ausgeführte matematische Behandlung seiner Vegetatiosversuche, inbesondere über den mathematischen Ausdruck des Setzes vom Minimum. Landwirtschaftliche Jahrbücher; vol. 42, pp. 425-428. 1912. 
8-PFEIFFER, Th., E. BLANCK e M. FLUGEL - Wasser und Licht als Vegetationsfaktoren und ihre Beziehungen zum Gesetze vom Minimum. Die landwirtschaftlichen Versuchs - Stationen, vol. LXXVI. Berlim. 1912.

9-MITSCHERLICH, Eilh. Alfred - Die Bestimmung des Düngerbedürfnisses des Bodens. Paul Parey. Berlim. 3a. edição. 1930.

10 - RUSSELL, E. John - Soil conditions and Plant Growth. Longmans, Green and Co. Londres. 7a. edição. 1942.

11 - MITSCHERLICH, Eilh Alfred - Die zweite Annäherung des Wirkungsgesetzes der Wachstumsfaktoren. Zeitschrift fur Pflanzenernährung, Düngung und Bodenkunde, vol. XII, parte científica, pp. 273-282. Berlim. 1928.

12 - NIKLAS, H. e M. MILLER - Bemerkungen zu den Beweisverfahren der Konstanz der Wirkungsfaktoren. Zeitschrift fur Pflanzenernahrung, Dungung und Bodenkunde, vol. XV, parte científica, pp. 193-196. Berlim. 1930.

13 - SARAIVA, Mario, Admar LOPES DA CRUZ e Carlos DEL NEGRO - Contribuição para o estudo dos methodos de Mitscherlich, Wiessmann e Neubauer. Rio de Janeiro. 1937.

14 - KLETSCHOWSKY, W. M. e P. A. SHELESNOW - Ueber Verschiebungen der Wirkungsfaktoren von Stickstoff und Phosphorüure. Landwirtschaftiliche Jahrbücher, vol. LXXIV, fasc. 3, pp. 353-404. Berlim. 1931.

15 - JORET, Georges - Recherches sur les Sols de Limons de la Picardie et leur Etat Actuel de Fertilité. 2.a parte, IX. Annales Agronomiques, pp. 178-185. Paris. 1932.

16 - DEMOLON. Albert - Croissance des Végétaux Cultivés. Dunod. Paris. 3a. edição, 1946.

17 - HOOVER, C. Dale e A. G. NORMAN - Applicability of the Mitscherlich Method to the Determination of Available Phosphate in some Iowa Soils. Soil Science, vol. 53, pp. 329-340. Baltimore. 1942.

18 - VANDECAVEYE, S. C. - Diagnostic Techniques for Soils and Crops. The American Potash Institute. Washington, D. C. 1948.

19-MOULTON, Forest Ray - Liebig and after Liebig The Science Press Printing Company, Lancaster, Pennsylvania. 1942.

20-MELLO MORAES, J. - Determinação da necessidade de adubação - método de Mitscherlich. Apostila. Piracicaba. 1947. 
21-MITSCHERLICH, Eilh. Alfred - Die pflanzenphysiologische Lösung der chemischen Bodenanalyse. Landwirtschaftliche Jahrbücher, vol. 58, pp. 601-617. Berlim. 1923.

22-BAULE, B. - Zu Mitscherlichs Gesetz der physiologischen Beziehungen. Landwirtschaftliche Jahrbücher, vol. 51, pp. 363-385.

23-MITSCHERLICH, Eilh. Alfred - Zum Gesetz des Minimum. Landwirtschaftliche Jahrbücher, vol. 42, pp. 423-424. Berlim. 1912.

24-GUNTHER, E. e K. SEIDEL - Düngungsversuche nach Mitscherlich an Schimmelpilzen und Sprosspilzen. Landwirtschaftliche Jahrbücher, vol. 65, pp. 109-181. Berlim. 1927.

25 - REINHOLD, Joh. - Mitscherlich Verfahren zur Bestimmung des Düngergehaltes des Bodens. Landwirtschaftliche Jahrbücher, vol. 65, pp. 877-892. Berlim. 1927.

26 - HANKINS, O. G. e Harry W. TITUS - Growth, Fattening, and Meat Production. Yearbook of Agriculture, 1939, pp. 450-468. Washington, D. C.

27 - OLSEN, S. R. e B. T. SHAW - Chemical, Mitscherlich, and Neubauer Methods for Determining Available Potassium in Relation to Crop Response to Potash Fertilization Journal of the American Society of Agronomy, vol. 35, pp. 1-9. Geneva, N. Y. 1943.

28- WILLCOX, O. W. - Interpretation of Olsen and Shaw's Field tests by the Mitscherlich - Baule Theorem and the Universal Yield Diagram. Journal of the American Society of Agronomy, vol. 35, pp. 454-459. Geneva, N. Y. 1943.

29 - MITSCHERLICH, Eilh. Alfred e W. U. BEHRENS - Zur Formulierung des Ertragsgesetzes. Zeitschrift für Pflanzenernährung, Düngung und Bodenkunde, vol. XV, parte científica, pp. 94-101. Berlim. 1930.

30-MAGISTAD, O. C. - A Comparison of Mitscherlich Trials on Hawaiian Soils in Germany and in the Territory of Hawaii. Journal of the American Society of Agronomy, vol. 30, pp. 692-698. Geneva, N. Y. 1938.

31-RAUTERBERG, E. - Uber die Beziehung zwischen Wachstumfaktor und Ertrag unter besonderer Berücksichtigung der Berechnung der Konstanten $\mathrm{A}$ und $\mathrm{c}$ in der logarithmischen Gleichung von Mitscherlich. Bodenkunde und Pflanzenernährung, vol. 14, pp. 10-28. Berlim. 1939.

32 - BOTELHO DA COSTA, J. V. - Valor e limitação da lei do efeito dos fatores de crescimento. Anais do Instituto Superior de Agronomia, vol. XIV, pp. 131-135. Lisboa. 1943. 
